\title{
LETTER
}

\section{Acute kidney injury: taking aim at colistin}

\author{
Shayan Rashid ${ }^{1 *}$, Lilian Saro-Nunez ${ }^{1}$, Akanksha Kumar ${ }^{2}$, Amit Patel $^{3}$ and Amay Parikh ${ }^{4}$ \\ See related research by Rocco et al., http://ccforum.com/content/17/4/R174
}

We appreciate the contribution of Rocco and colleagues [1], whose retrospective study in a recent issue of Critical Care evaluated risk factors for acute kidney injury (AKI) in patients receiving colistin methanesulfonate (CMS) or other nephrotoxic antimicrobials. The following key thoughts occurred to us after reviewing the study.

We would like to address the criteria the authors used to qualify AKI. The authors note using RIFLE (Risk, Injury, and Failure; and Loss, and End-stage kidney disease) criteria to assess kidney injury, and note the degree of increase of serum creatinine from baseline in categorizing patients. However, the authors do not comment on urine output in these patients. Both urine output and serum creatinine are critical variables in recognizing kidney injury in a timely manner [2].

As noted, the literature on CMS pharmacokinetics is limited. A recent study investigating the pharmacokinetics of colistin noted that the maximum plasma concentration-to-minimum inhibitory concentration was much lower for Pseudomonas species than for other Gram-negative rods [3]. This implies that higher doses of CMS, and potentially more nephrotoxic doses, are required for treating Pseudomonas infections. Additionally, for those patients receiving continuous renal replacement therapy, the effective dose of antibiotic received may vary. It would be interesting to see what relationship, if any, exists between AKI and targeted bacteria species in critically ill patients on CMS.

We appreciate the contribution of the authors to the literature on the prediction of AKI in critically ill patients receiving nephrotoxic antimicrobials. Further investigations into both the efficacy and potential harm of this antimicrobial are warranted.
Abbreviations

AKI: Acute kidney injury; CMS: Colistin methanesulfonate.

Competing interests

The authors declare that they have no competing interests.

\section{Author details}

${ }^{1}$ Internal Medicine Division, Department of Medicine, Rutgers Robert Wood Johnson Medical School, 1 Robert Wood Johnson Place, P.O. Box 19, New Brunswick, NJ 08903-0019, USA. ${ }^{2}$ Department of Family Medicine and Community Health, Department of Medicine, Rutgers Robert Wood Johnson Medical School, 1 Robert Wood Johnson Place, PO Box 19, New Brunswick, NJ 08903-0019, USA. ${ }^{3}$ Pulmonary and Critical Care Division, Department of Medicine, Rutgers Robert Wood Johnson Medical School, 1 Robert Wood

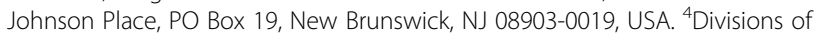
Nephrology and Pulmonary/Critical Care, Department of Medicine, Rutgers Robert Wood Johnson Medical School, 1 Robert Wood Johnson Place, PO Box 19, New Brunswick, NJ 08903-0019, USA.

Published: 21 Jan 2014

\section{References}

1. Rocco M, Montini L, Alessandri E, Venditti M, Laderchi A, De Pascale G, Raponi G, Vitale M, Pietropaoli P, Antonelli M: Risk factors for acute kidney injury in critically ill patients receiving high intravenous doses of colistin methanesulfonate and/or other nephrotoxic antibiotics: a retrospective cohort study. Crit Care 2013, 17:R174.

2. Bellomo R, Ronco C, Kellum JA, Mehta RL, Palevsky P: Acute renal failure definition, outcome measures, animal models, fluid therapy and information technology needs: the Second International Consensus Conference of the Acute Dialysis Quality Initiative (ADQI) Group. Crit Care 2004, 8:R204-R212.

3. Karnik ND, Sridharan K, Jadhav SP, Kadam PP, Naidu RK, Namjoshi RD, Gupta V, Gore MS, Surase PV, Mehta PR, Gogtay JA, Thatte UM, Gogtay NJ: Pharmacokinetics of colistin in critically ill patients with multidrug-resistant Gram-negative bacilli infection. Eur J Clin Pharmacol 2013, 69:1429-1436.

$10.1186 / \mathrm{cc} 13703$

Cite this article as: Rashid et al:: Acute kidney injury: taking aim at colistin. Critical Care 2014, 18:401

\footnotetext{
* Correspondence: srashid88@gmail.com

'Internal Medicine Division, Department of Medicine, Rutgers Robert Wood Johnson Medical School, 1 Robert Wood Johnson Place, P.O. Box 19, New Brunswick, NJ 08903-0019, USA

Full list of author information is available at the end of the article
} 